\title{
Contribution à l'étude de la flore levure du fromage de Camembert (II)
}

\author{
par \\ J. L. SchMidT et J. LENOIR* \\ (avec la collaboration technique de Michèle ScHmIDT)
}

\section{INTRODUCTION}

Dans une publication antérieure, nous avons souligné l'importance de la flore levure dans les fromages à pâte molle de type Camembert (Schmidt et Lenoir [11]). Les fromages étudiés étaient fabriqués à partir de lait cru et provenaient de cinq usines de Normandie choisies pour la qualité et la régularité de leur production.

Les résultats obtenus ont mis en évidence une population de levures importante, en surface du fromage, dès les premiers jours de fabrication (4.10 à $2.10^{8}$ par gramme le quatrième jour), cette population proliférant activement jusqu'aux environs du dixième jour $\left(5.10^{8}\right.$ à $\left.3.10^{9}\right)$. A l'intérieur du fromage, les levures se développent sensiblement moins, et il faut attendre le quatorzième jour pour noter une population de $2.10^{5}$ à $1.10^{7}$ par gramme de pâte. D'une usine à l'autre, les profils représentant l'évolution des levures sont relativement similaires ; en revanche, il a été observé que leur nombre pouvait être variable.

L'identification de cette flore a été pratiquée sur les prélèvements provenant de trois usines, l'importance relative des diverses espèces est variable selon l'origine.

Cependant, la flore levure du Camembert était dominée par le groupe des Kluyveromyces, Kl. Lactis, Kl. fragilis et leurs formes imparfaites, Torulopsis sphaerica et Candida pseudotropicalis, qui représentent plus de 60 p. 100 de la population. Venaient ensuite les espèces Debaryomyces hansenii (12 p. 100), Saccharomyces cerevisiae (9 p.100), Saccharomyces rouxii $(6 \mathrm{p} .100)$ et Torulopsis versatilis (4 p. 100).

* Laboratoire de Recherches de la Chaire de Technologie (I.N.R.A.), Institut National Agronomique Paris-Grignon - 78850 Thiverval-Grignon. 
La présente étude prolonge la précédente ; elle vise, en disposant d'un échantillonnage plus large et plus représentatif, à préciser l'identité des espèces majeures de levures présentes à la surface et à l'intérieur du fromage au cours de la maturation.

Cent quatre-vingt-dix-neuf souches, isolées à partir de fabrications issues de deux nouvelles usines, ont été étudiées, et cent quatre-vingttreize souches ont pu être identifiées.

L'ensemble de la collection de levures du laboratoire compte à présent quatre cent quatre-vingt-deux souches identifiées sur cinq cent vingt-trois isolées.

Les fromages étudiés proviennent de deux grandes régions laitières de Normandie, le Pays d'Auge (région A : séries 1, 3 et 5 ) et la région d'Isigny-sur-Mer (région B : séries 2 et 4 ).

\section{MATERIELS ET METHODES}

Les méthodes de dénombrement et d'isolement des souches prélevées à divers stades de l'affinage ont été précédemment décrites (Schmidt et Lenoir, [11]).

L'identification des espèces a été conduite sur la base des classifications de Lodder [8] et de Barnett et Pankhurst [1]. Les caractères culturaux, morphologiques, biochimiques et physiologiques étudiés sont analogues à ceux mentionnés dans notre note antérieure.

\section{RESULTATS}

\section{Identification de la flore levure}

Dans les deux fabrications analysées (4 et 5) les espèces suivantes ont été identifiées :

- Saccharomyces italicus Castelli;

- Kluyveromyces lactis (Dombrowski) Van der Walt et sa forme imparfaite Torulopsis sphaerica (Hammer et Cordes) Lodder;

- Debaryomyces hansenii (Zopf) Lodder et Kreger Van Rij ;

- Kluyveromyces fragilis (Jörgensen) Van der Walt et sa forme asexuée Candida pseudotropicalis (Castellani) Basgal ;

- Kluyveromyces bulgaricus (Santa Maria) Van der Walt.

La répartition des espèces entre les deux fabrications figure sur le tableau 1. Comme il a été précédemment noté, on observe que l'importance relative des diverses espèces est variable selon la fabrication. Ainsi Kluyveromyces lactis et Torulopsis sphaerica représentent 35 p. 100 de la population dans la fabrication 4, seulement 


\section{TABLEAU 1}

Répartition des principales espèces de levures isolées des fromages des deux fabrications

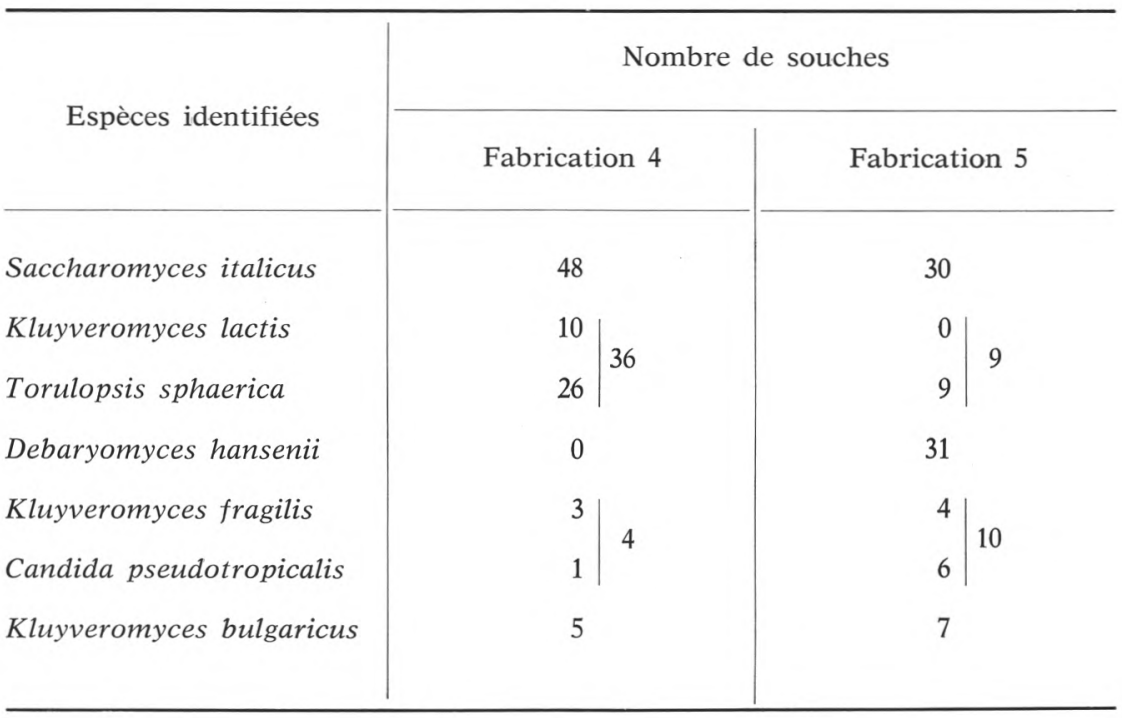

\section{TABLEAU 2}

Espèces de levures trouvées en faible nombre dans les fromages de deux fabrications

\begin{tabular}{l|c}
\hline \multicolumn{1}{c|}{ Espèces identifiées } & Nombre de souches \\
\hline Torulopsis versatilis & 3 \\
Torulopsis bovina & 3 \\
Candida lambica & 2 \\
Candida valida & 2 \\
Saccharomyces cerevisiae & 1 \\
Torulopsis holmii & 1 \\
Rhodotorula sp. & 1 \\
\end{tabular}


10 p. 100 dans la fabrication 5 ; Debaryomyces hansenii, absent en 4, est présent au taux de 34 p. 100 en 5.

A côté de ces espèces dominantes, qui regroupent cent-quatrevingts souches, treize souches appartiennent à sept espèces différentes (tab. 2).

\section{Evolution qualitative de la flore levure}

La répartition des différentes espèces de levures au cours de la maturation des fromages des deux fabrications est donnée dans les tableaux 3 et 4 .

\section{TABLEAU 3}

Répartition des espèces au cours de l'affinage des fromages de la fabrication 4

\begin{tabular}{|c|c|c|c|c|c|c|}
\hline \multirow{3}{*}{\multicolumn{2}{|c|}{ Espèces }} & \multicolumn{4}{|c|}{ Stades de maturation (jours) } & \multirow{3}{*}{$\begin{array}{c}\text { p. } 100 \\
\text { du } \\
\text { prélève- } \\
\text { ment } \\
\text { total }\end{array}$} \\
\hline & & \multicolumn{2}{|c|}{$1-8 j$} & \multicolumn{2}{|c|}{$13-32 j$} & \\
\hline & & $\begin{array}{l}\text { Nombre } \\
\text { de } \\
\text { souches }\end{array}$ & $\begin{array}{l}\text { p. } 100 \mathrm{du} \\
\text { prélève- } \\
\text { ment }\end{array}$ & $\begin{array}{l}\text { Nombre } \\
\text { de } \\
\text { souches }\end{array}$ & $\begin{array}{l}\text { p. } 100 \mathrm{du} \\
\text { prélève- } \\
\text { ment }\end{array}$ & \\
\hline Saccharomyces italicus & $\begin{array}{l}\mathrm{E} \\
\mathrm{I}\end{array}$ & $\begin{array}{l}4 \\
7\end{array}$ & $\begin{array}{l}14 \\
32\end{array}$ & $\begin{array}{l}13 \\
24\end{array}$ & $\begin{array}{l}72 \\
73\end{array}$ & $\begin{array}{l}36 \\
56\end{array}$ \\
\hline $\begin{array}{l}\text { Kluyveromyces lactis } \\
\text { et Torulopsis sphaerica }\end{array}$ & $\begin{array}{l}\mathrm{E} \\
\mathrm{I}\end{array}$ & $\begin{array}{l}16 \\
12\end{array}$ & $\begin{array}{l}55 \\
54,5\end{array}$ & $\begin{array}{l}4 \\
4\end{array}$ & $\begin{array}{l}22 \\
12\end{array}$ & $\begin{array}{l}42,5 \\
29\end{array}$ \\
\hline Kluyveromyces bulgaricus & $\begin{array}{l}E \\
I\end{array}$ & $\begin{array}{l}3 \\
1\end{array}$ & $\begin{array}{c}10 \\
4,5\end{array}$ & $\begin{array}{l}0 \\
1\end{array}$ & $\begin{array}{l}0 \\
3\end{array}$ & $\begin{array}{l}6 \\
3,5\end{array}$ \\
\hline $\begin{array}{l}\text { Kluyveromyces fragilis et } \\
\text { Candida pseudotropicalis }\end{array}$ & $\begin{array}{l}E \\
I\end{array}$ & $\begin{array}{l}2 \\
1\end{array}$ & $\begin{array}{l}7 \\
4,5\end{array}$ & $\begin{array}{l}1 \\
0\end{array}$ & $\begin{array}{c}5,5 \\
0\end{array}$ & $\begin{array}{l}6 \\
2\end{array}$ \\
\hline Torulopsis versatilis & $\begin{array}{l}E \\
I\end{array}$ & $\begin{array}{l}0 \\
0\end{array}$ & $\begin{array}{l}0 \\
0\end{array}$ & $\begin{array}{l}0 \\
3\end{array}$ & $\begin{array}{l}0 \\
9\end{array}$ & $\begin{array}{l}0 \\
5,5\end{array}$ \\
\hline Espèces diverses & $\begin{array}{l}E \\
I\end{array}$ & $\begin{array}{l}4 \\
1\end{array}$ & $\begin{array}{c}14 \\
4,5\end{array}$ & $\begin{array}{l}0 \\
1\end{array}$ & $\begin{array}{l}0 \\
3\end{array}$ & $\begin{array}{l}8,5 \\
3,5\end{array}$ \\
\hline $\begin{array}{l}\text { E : Extérieur du fromage } \\
\text { I : Intérieur du fromage. }\end{array}$ & & & & & & \\
\hline
\end{tabular}


TABLEAU 4

Répartition des espèces au cours de l'affinage des fromages de la fabrication 5

\begin{tabular}{|c|c|c|c|c|c|c|}
\hline \multirow{3}{*}{ Espèces } & & \multicolumn{4}{|c|}{ Stades de maturation (jours) } & \multirow{3}{*}{$\begin{array}{l}\text { p. } 100 \mathrm{du} \\
\text { du } \\
\text { prélève- } \\
\text { ment } \\
\text { total }\end{array}$} \\
\hline & & \multicolumn{2}{|c|}{$1-8 j$} & \multicolumn{2}{|c|}{$13-32 j$} & \\
\hline & & $\begin{array}{l}\text { Nombre } \\
\text { de } \\
\text { souches }\end{array}$ & $\begin{array}{c}\text { p. } 100 \text { du } \\
\text { prélève- } \\
\text { ment }\end{array}$ & $\begin{array}{c}\text { Nombre } \\
\text { de } \\
\text { souches }\end{array}$ & $\begin{array}{l}\text { p. } 100 \text { du } \\
\text { prélève- } \\
\text { ment }\end{array}$ & \\
\hline Debaryomyces hansenii & $\begin{array}{l}E \\
I\end{array}$ & $\begin{array}{l}11 \\
14\end{array}$ & $\begin{array}{l}41 \\
48,5\end{array}$ & $\begin{array}{l}6 \\
0\end{array}$ & $\begin{array}{r}40 \\
0\end{array}$ & $\begin{array}{l}40,5 \\
25,5\end{array}$ \\
\hline Saccharomyces italicus & $\begin{array}{l}E \\
I\end{array}$ & $\begin{array}{l}4 \\
3\end{array}$ & $\begin{array}{l}15 \\
10,5\end{array}$ & $\begin{array}{r}2 \\
21\end{array}$ & $\begin{array}{l}13,5 \\
81\end{array}$ & $\begin{array}{l}14,5 \\
43,5\end{array}$ \\
\hline $\begin{array}{l}\text { Kluyveromyces fragilis et } \\
\text { Candida pseudotropicalis }\end{array}$ & $\begin{array}{l}E \\
I\end{array}$ & $\begin{array}{l}5 \\
2\end{array}$ & $\begin{array}{c}18,5 \\
7\end{array}$ & $\begin{array}{l}2 \\
1\end{array}$ & $\begin{array}{c}13,5 \\
4\end{array}$ & $\begin{array}{r}16,5 \\
5,5\end{array}$ \\
\hline Torulopsis sphaerica & $\begin{array}{l}E \\
I\end{array}$ & $\begin{array}{l}1 \\
4\end{array}$ & $\begin{array}{r}4 \\
14\end{array}$ & $\begin{array}{l}4 \\
0\end{array}$ & $\begin{array}{r}27 \\
0\end{array}$ & 12 \\
\hline Kluyveromyces bulgaricus & $\begin{array}{l}E \\
\mathrm{I}\end{array}$ & $\begin{array}{l}2 \\
3\end{array}$ & $\begin{array}{r}7,5 \\
10,5\end{array}$ & $\begin{array}{l}1 \\
1\end{array}$ & $\begin{array}{l}6,5 \\
4\end{array}$ & $\begin{array}{l}7 \\
7,5\end{array}$ \\
\hline Espèces diverses & $\begin{array}{l}E \\
\mathrm{I}\end{array}$ & $\begin{array}{l}1 \\
1\end{array}$ & $\begin{array}{l}4 \\
3,5\end{array}$ & $\begin{array}{l}0 \\
2\end{array}$ & $\begin{array}{l}0 \\
8\end{array}$ & $\begin{array}{l}2,5 \\
5,5\end{array}$ \\
\hline \multicolumn{7}{|l|}{$\begin{array}{l}\text { E : Extérieur du fromage. } \\
\text { I : Intérieur du fromage. }\end{array}$} \\
\hline
\end{tabular}

Il apparaît que, dans la fabrication 4, deux espèces dominent. Saccharomyces italicus, déjà présente en début d'affinage à l'intérieur de la pâte (32 p. 100), forme, dans la seconde phase de la maturation, la majeure partie de la flore levure, tant à la surface (72 p.100) qu'à l'intérieur du fromage (73 p. 100). Kluyveromyces lactis et sa forme anascosporogène, Torulopsis sphaerica, sont également bien représentées en début d'affinage, que ce soit sur les parties superficielles ou internes de la pâte (55 p. 100), et elles conservent une certaine implantation du treizième au trentième jour de maturation. En revanche, Kluyveromyces fragilis est peu abondante et l'espèce Debaryomyces hansenii est absente. 
TABLEAU 5

Les principales espèces de levures présentes au cours de l'affinage des fromages selon les fabrications (en p. 100 du prélèvement)

\begin{tabular}{|c|c|c|c|c|c|c|c|c|c|c|c|}
\hline \multirow{3}{*}{ Espèces } & & \multicolumn{10}{|c|}{ Stades de maturation (jours) } \\
\hline & & \multicolumn{5}{|c|}{$1-8 j$} & \multicolumn{5}{|c|}{$13-32 j$} \\
\hline & & 1 & 2 & 3 & 4 & 5 & 1 & 2 & 3 & 4 & 5 \\
\hline $\begin{array}{l}\text { Kluyveromyces lactis } \\
\text { et Torulopsis sphaerica }\end{array}$ & $\begin{array}{l}E \\
I\end{array}$ & $\begin{array}{l}7 \\
3\end{array}$ & $\begin{array}{l}56 \\
38,5\end{array}$ & $\begin{array}{l}37,5 \\
21\end{array}$ & $\begin{array}{l}55 \\
54,5\end{array}$ & $\begin{array}{r}4 \\
14\end{array}$ & $\begin{array}{l}63 \\
17\end{array}$ & $\begin{array}{l}19 \\
19\end{array}$ & $\begin{array}{l}95 \\
80\end{array}$ & $\begin{array}{l}22 \\
12\end{array}$ & $\begin{array}{r}27 \\
0\end{array}$ \\
\hline Saccharomyces italicus & $\begin{array}{l}E \\
l\end{array}$ & $\begin{array}{l}0 \\
6\end{array}$ & $\begin{array}{l}0 \\
0\end{array}$ & $\begin{array}{l}0 \\
0\end{array}$ & $\begin{array}{l}14 \\
32\end{array}$ & $\begin{array}{l}15 \\
10,5\end{array}$ & $\begin{array}{l}0 \\
0\end{array}$ & $\begin{array}{l}0 \\
0\end{array}$ & $\begin{array}{l}0 \\
0\end{array}$ & $\begin{array}{l}72 \\
73\end{array}$ & $\begin{array}{l}13,5 \\
81\end{array}$ \\
\hline $\begin{array}{l}\text { Kluyveromyces fragilis } \\
\text { et Candida pseudotropicalis }\end{array}$ & $\begin{array}{l}E \\
I\end{array}$ & $\begin{array}{r}52 \\
6\end{array}$ & $\begin{array}{l}12,5 \\
42\end{array}$ & $\begin{array}{c}12,5 \\
0\end{array}$ & $\begin{array}{l}7 \\
4,5\end{array}$ & $\begin{array}{c}18,5 \\
7\end{array}$ & $\begin{array}{l}4 \\
8\end{array}$ & $\begin{array}{l}43 \\
46\end{array}$ & $\begin{array}{l}0 \\
0\end{array}$ & $\begin{array}{l}5,5 \\
0\end{array}$ & $\begin{array}{c}13,5 \\
4\end{array}$ \\
\hline $\begin{array}{l}\text { Debaryomyces hensenii } \\
\text { et Torulopsis candida }\end{array}$ & $\begin{array}{l}E \\
I\end{array}$ & $\begin{array}{l}3,5 \\
9\end{array}$ & $\begin{array}{l}9 \\
8\end{array}$ & $\begin{array}{l}9 \\
7\end{array}$ & $\begin{array}{l}0 \\
0\end{array}$ & $\begin{array}{l}41 \\
48,5\end{array}$ & $\begin{array}{l}18,5 \\
58\end{array}$ & $\begin{array}{l}5 \\
4\end{array}$ & $\begin{array}{l}5 \\
0\end{array}$ & $\begin{array}{l}0 \\
0\end{array}$ & $\begin{array}{r}40 \\
0\end{array}$ \\
\hline Saccharomyces cerevisiae & $\begin{array}{l}\mathrm{E} \\
\mathrm{I}\end{array}$ & $\begin{array}{l}14 \\
56\end{array}$ & $\begin{array}{l}0 \\
0\end{array}$ & $\begin{array}{l}0 \\
3,5\end{array}$ & $\begin{array}{l}0 \\
4,5\end{array}$ & $\begin{array}{l}0 \\
0\end{array}$ & $\begin{array}{l}0 \\
4\end{array}$ & $\begin{array}{l}5 \\
0\end{array}$ & $\begin{array}{l}0 \\
0\end{array}$ & $\begin{array}{l}0 \\
0\end{array}$ & $\begin{array}{l}0 \\
0\end{array}$ \\
\hline $\begin{array}{l}\text { Saccharomyces rouxii } \\
\text { et Torulopsis mogii }\end{array}$ & $\begin{array}{l}\mathrm{E} \\
\mathrm{I}\end{array}$ & $\begin{array}{l}0 \\
8\end{array}$ & $\begin{array}{l}0 \\
0\end{array}$ & $\begin{array}{r}9 \\
41\end{array}$ & $\begin{array}{l}0 \\
0\end{array}$ & $\begin{array}{l}0 \\
0\end{array}$ & $\begin{array}{l}0 \\
0\end{array}$ & $\begin{array}{l}0 \\
0\end{array}$ & $\begin{array}{l}0 \\
0\end{array}$ & $\begin{array}{l}0 \\
0\end{array}$ & $\begin{array}{l}0 \\
0\end{array}$ \\
\hline Torulopsis versatilis & $\begin{array}{l}\mathrm{E} \\
\mathrm{I}\end{array}$ & $\begin{array}{l}7 \\
3\end{array}$ & $\begin{array}{l}3 \\
0\end{array}$ & $\begin{array}{l}0 \\
7\end{array}$ & $\begin{array}{l}0 \\
0\end{array}$ & $\begin{array}{l}0 \\
0\end{array}$ & $\begin{array}{l}4 \\
4\end{array}$ & $\begin{array}{c}5 \\
11,5\end{array}$ & $\begin{array}{l}0 \\
4\end{array}$ & $\begin{array}{l}0 \\
9\end{array}$ & $\begin{array}{l}0 \\
0\end{array}$ \\
\hline Kluyveromyces bulgaricus & $\begin{array}{l}E \\
\mathrm{I}\end{array}$ & $\begin{array}{l}0 \\
0\end{array}$ & $\begin{array}{l}0 \\
0\end{array}$ & $\begin{array}{l}3 \\
0\end{array}$ & $\begin{array}{c}10 \\
4,5\end{array}$ & $\begin{array}{r}7,5 \\
10,5\end{array}$ & $\begin{array}{l}0 \\
0\end{array}$ & $\begin{array}{l}0 \\
0\end{array}$ & $\begin{array}{l}0 \\
0\end{array}$ & $\begin{array}{l}0 \\
3\end{array}$ & $\begin{array}{l}7 \\
7,5\end{array}$ \\
\hline Espèces diverses & $\begin{array}{l}E \\
I\end{array}$ & $\begin{array}{c}16,5 \\
9\end{array}$ & $\begin{array}{l}12,5 \\
11,5\end{array}$ & $\begin{array}{l}6 \\
0\end{array}$ & $\begin{array}{r}14 \\
0\end{array}$ & $\begin{array}{l}4 \\
3,5\end{array}$ & $\begin{array}{c}10,5 \\
9\end{array}$ & $\begin{array}{l}9,5 \\
4\end{array}$ & $\begin{array}{l}0 \\
0\end{array}$ & $\begin{array}{l}0 \\
3\end{array}$ & $\begin{array}{l}0 \\
8\end{array}$ \\
\hline
\end{tabular}

1, 2, 3, 4, 5 : Numéro d'ordre des différentes fabrications.

E : Extérieur du fromage; I : Intérieur du fromage. 
Dans la fabrication 5, la diversité des espèces est plus grande. Debaryomyces hansenii domine à la surface $(41$ p. 100) et à l'intérieur de la Pâte (48 p. 100) dans les premiers stades mais, du treizième au trente-deuxième jour, elle ne subsiste plus qu'en surface. L'espèce Saccharomyces italicus, faiblement implantée en début d'affinage, devient très largement dominante en fin de maturation au sein de la pâte (81 p. 100). Kluyveromyces fragilis et sa forme imparfaite, Candida pseudotropicalis, sont essentiellement présentes à la partie superficielle dans les deux phases de l'affinage (18 et 13 p. 100). Enfin, Kluyveromyces lactis, qui n'est représentée que par sa forme asexuée, Torulopsis sphaerica, apparaît, dans la seconde phase, localisée en surface (27 p. 100).

\section{Récapitulation de la flore levure du Camembert}

Le tableau 5 présente les principales espèces de levures rencontrées au cours de l'affinage des fromages dans les cinq fabrications que nous avons étudiées. On peut ainsi remarquer que les deux principaux groupes, Kluyveromyces et Debaryomyces se retrouvent pratiquement dans toutes les fabrications, mais leur répartition peut être différente d'une série à l'autre. En ce qui concerne les trois espèces de Saccharomyces identifiées, il apparaît que leur implantation, qui peut être massive, n'est réalisée que dans certaines fabrications et plutôt à l'intérieur de la pâte.

On peut finalement relever (tab. 6) que Kluyveromyces lactis et sa forme imparfaite Torulopsis sphaerica sont les espèces majeures de la flore levure du Camembert ( 34 p. 100 de la population). Se situent ensuite, et sensiblement au même seuil voisin de 15 p. 100, les espèces Saccharomyces italicus, Kluyveromyces fragilis et Candida pseudotropicalis, Debaryomyces hansenii et Torulopsis candida. Enfin, un dernier groupe est constitué de Saccharomyces cerevisiae, Saccharomyces rouxii, Torulopsis versatilis et Kluyveromyces bulgaricus, chacune de ces espèces représentant 3 à 5 p. 100 de la flore levure totale.

\section{DISCUSSION}

Cette étude taxonomique confirme les premiers résultats que nous avions enregistrés (Schmidt et Lenoir, [11]). En effet, dans les deux nouvelles fabrications analysées (4 et 5 ), se retrouve la même diversité de flore que dans les fabrications déjà étudiées (1 à 3). Ainsi, Kluyveromyces lactis et Torulopsis sphaerica sont constamment présentes (tab. 3 et 4). Debaryomyces hansenii est bien représentée dans l'une des séries, alors que Kluyveromyces fragilis et Candida pseudotropicalis ne sont que faiblement implantées. Saccharomyces italicus, espèce majeure des fabrications 4 et 5 , n'avait été jusqu'ici trouvée qu'en petit nombre. On peut cependant observer que cette 


\section{TABLEAU 6}

Identité des espèces de levures présentes dans les cinq fabrications de fromages de Camembert étudiés

\begin{tabular}{|c|c|c|c|c|}
\hline Espèces identifiées & \multicolumn{2}{|c|}{ Nombre de souches } & \multicolumn{2}{|c|}{$\begin{array}{l}\text { p. } 100 \text { des souches } \\
\text { identifiées }\end{array}$} \\
\hline $\begin{array}{l}\text { Kluyveromyces lactis } \\
\text { Torulopsis sphaerica }\end{array}$ & $\begin{array}{r}58 \\
105\end{array}$ & 163 & $\begin{array}{l}12 \\
21,8\end{array}$ & 33,8 \\
\hline Saccharomyces italicus & & 80 & & 16,6 \\
\hline $\begin{array}{l}\text { Kluyveromyces fragilis } \\
\text { Candida pseudotropicalis }\end{array}$ & $\begin{array}{l}49 \\
25\end{array}$ & 74 & $\begin{array}{r}10,2 \\
5,2\end{array}$ & 15,4 \\
\hline $\begin{array}{l}\text { Debaryomyces hansenii } \\
\text { Torulopsis candida }\end{array}$ & $\begin{array}{r}65 \\
2\end{array}$ & 67 & $\begin{array}{r}13,5 \\
0,4\end{array}$ & 13,9 \\
\hline Saccharomyces cerevisiae & & 26 & & 5,4 \\
\hline $\begin{array}{l}\text { Saccharomyces rouxii } \\
\text { Torulopsis mogii }\end{array}$ & $\begin{array}{r}8 \\
10\end{array}$ & 18 & $\begin{array}{l}1,7 \\
2,1\end{array}$ & 3,7 \\
\hline Torulopsis versatilis & & 16 & & 3,3 \\
\hline Kluyveromyces bulgaricus & & 13 & & 2,7 \\
\hline Espèces diverses & & 25 & & 5,2 \\
\hline Total & & 482 & & 100 \\
\hline
\end{tabular}

espèce est considérée par Lodder [8] comme très proche de Saccharomyces cerevisiae et les réponses aux principaux critères d'identification, caractères culturaux et morphologiques, aptitude à la formation d'ascospores et de filaments mycéliens, sont très voisins. Selon la grille d'identification de Barnett et Pankhurst [1], les différences entre les deux espèces reposent principalement sur les tests d'assimilation et de fermentation du raffinose; mais, ces auteurs considèrent que les validités de ces deux tests sont relativement faibles (respectivement 27 et 6,4 p. 100 par rapport à la validité du test d'assimilation du maltose prise comme référence $=100$ ). Une parenté aussi étroite autorise à classer les deux espèces dans un groupe unique, et la récente classification des levures proposées par Barnett et al. [2] les regroupe sous le nom de Saccharomyces cerevisiae.

Une autre espèce rencontrée dans les fabrications 4 et 5 , en proportion non négligeable, est Kluyveromyces bulgaricus. Elle s'appa- 


\section{TABLEAU 7}

Répartition des principales espèces de levures isolées des fromages de différentes fabrications (en p. 100 du prélèvement)

\begin{tabular}{|c|c|c|c|c|c|}
\hline Espèces identifiées & 1 & 2 & 3 & 4 & 5 \\
\hline $\begin{array}{l}\text { K1. Lactis / T. sphaerica } \\
\text { Kl. fragilis / C. pseudotropicalis } \\
\text { et Kl. bulgaricus }\end{array}$ & 41,9 & 78,7 & 67,8 & 44,1 & 28,6 \\
\hline $\begin{array}{l}\text { D. hansenii } \\
\text { S. italicus et S. cerevisiae }\end{array}$ & 45,7 & 8,5 & 7,8 & 48 & 67 \\
\hline S. rouxii / T. mogii & 1,9 & 0 & 17,8 & 0 & 0 \\
\hline T. versatilis & 4,8 & 5,3 & 3,3 & 2,9 & 0 \\
\hline Espèces diverses & 5,7 & 7,4 & 3,3 & 4,9 & 4,4 \\
\hline Total des souches identifiées & 105 & 94 & 90 & 102 & 91 \\
\hline
\end{tabular}

$1,2,3,4$ et 5 : Numéro d'ordre des différentes fabrications.

rente, au plan physiologique, à Kluyveromices fragilis; elle s'en distingue essentiellement par la forme des ascospores et elle avait été décrite par Santa Maria [10] comme Saccharomyces fragilis var. bulgaricus.

Si l'on envisage la répartition des espèces suivant, d'une part, le stade d'affinage et, d'autre part, les parties internes ou superficielles du fromage, on observe que, dans l'ensemble, Saccharomyces italicus est plus nettement dominante en fin d'affinage. Pour les autres espèces, la répartition paraît variable selon les fabrications et aucune règle ne peut être dégagée. Debaryomyces hansenii, par exemple, est présente dans une série, tant à l'intérieur qu'à l'extérieur, au stade 1-8 $\mathrm{j}$, et seulement à la surface du fromage au stade 13-32 j, alors qu'elle est absente dans la seconde série.

En considérant l'ensemble des résultats des identifications effectuées sur les cinq fabrications étudiées (tab. 5), il paraît donc difficile de dégager de grandes tendances dans l'implantation, en surface ou à l'intérieur du fromage et, selon le stade de maturation, d'une espèce ou même d'un groupe d'espèces apparentées.

Compte tenu des différences des milieux naturels des deux régions concernées et des caractères de " cru » de leurs produits laitiers, 
il était permis de penser que les flores levures locales présenteraient, chacune, des profils originaux. A l'analyse du tableau 5, la notion de " niche levurienne " ne paraît pas évidente.

Les principales espèces identifiées entrent dans les groupes qui avaient été partiellement reconnus par Lenoir et Auberger [7] dans leur étude sur la flore du Camembert. Ce sont également celles qui ont été le plus fréquemment rencontrées dans le Saint-Nectaire (Dale [3] ; Vergeade et al. [12]), à l'intérieur du Roquefort (Devoyod et Sponem [4]; Galzin et al. [6], dans le Saint-Paulin (Ducastelle et Lenoir [5]) et dans le Cantal (Millet et al. [9]).

Finalement, en se référant aux résultats obtenus sur les cinq fabrications étudiées et en regroupant les espèces apparentées, on peut remarquer que la population en levures du Camembert paraît être constituée " d'une flore de fond » à laquelle appartient un petit nombre d'espèces du groupe Kluyveromyces (tab. 7). A côté de cette flore, non toujours dominante, on relève ce que l'on peut appeler des « touches » particulières, apportées par l'importance relative du groupe Debaryomyces-Saccharomyces et, parfois, la présence d'une espèce donnée, telle Saccharomyces rouxii.

\title{
Résumé
}

La nature de la flore levure du fromage de Camembert a été précisée sur deux nouvelles fabrications. Cent quatre-vingt-treize souches ont été isolées et identifiées selon les critères préconisés par Lodder, Barnett et Pankhurst. Dans ces fabrications, cette flore paraît être dominée par Saccharomyces italicus ainsi que par Kluyveromyces lactis et sa forme imparfaite Torulopsis sphaerica. L'espèce Debaryomyces hansenii est également bien représentée.

Finalement, après analyse de la flore levure de cinq fabrications et identification de quatre cent quatre-vingt-deux souches prélevées à différents stades de l'affinage, les espèces principalement rencontrées, malgré les variations que l'on peut constater d'une série à l'autre, sont $K l$. lactis $/ T$. sphaerica, Kl. fragilis $/ C$. pseudotropicalis, D. hansenii ainsi que le groupe des Saccharomyces ( $S$. italicus et $S$. cerevisiae).

\section{S u m mary}

\author{
STUDY OF THE YEAST FLORA COMPOSITION \\ OF CAMEMBERT CHEESE
}

The yeast flora composition of Camembert cheese was precised from two new manufacturings.

193 strains were isolated and their identification was made according to the criteria recommended by Lodder and by Barnett and Pankhurst. 
In these cheesemakings, the yeast flora seemed to be dominated by $S$. italicus and also by $K l$. lactis and its imperfect form $T$. sphaerica. The species $D$. hansenii is also well represented.

Finally, after analysis of the yeast flora from five different lots, 482 strains, sampled at different periods of ripening, were identified.

The main species found, despite fluctuations from one production to another, were $K l$. lactis $/ T$. sphaerica, Kl. fragilis $/ C$. pseudotropicalis, D. hansenii, as Saccharomyces group (S. italicus and $S$. cerevisiae).

Reçu pour publication en mars 1980.

\section{Bibliographie}

[1] Barnett (J. A.) and Pankhurst (R. J.) (1974). - A new key to the yeasts. North Holland Publishing Company, Amsterdam-London.

[2] Barnett (J. A.), Payne (R. W.) and Yarrow (D.) (1979). - A guide to identifying and classifying yeasts. Cambridge University Press, Cambridge.

[3] Dale (G.) (1972). - Moisissures levures du fromage de Saint-Nectaire. Rev. Lait. Fr., 296, 199.

[4] Devoyod (J. J.) et Sponem (D.) (1970). - La flore microbienne du fromage de Roquefort. VI. Les levures. Le Lait, 498, 524.

[5] Ducastelle (A.) et Lenorr (J.) (1965). - Contribution à l'étude de la flore microbienne du fromage de type Saint-Paulin. II. Ses espèces dominantes. Le Lait, 45 (448), 509.

[6] Galzin (M.), Galzy (P.) et Bret (G.) (1970). - Etude de la flore de levure dans les fromages de Roquefort. Le Lait, 50 (491-492), 1.

[7] Lenoir (J.) et Auberger (B.) (1966). - Contribution à l'étude de la flore microbienne du fromage de type Camembert. C.R. XVII e Cong. Int. Lait., D 395, 595.

[8] LodDer (J.) (1971). - The yeasts. A taxonomic study. North Holland Plublishing Company, Amsterdam-London.

[9] Millet (L.), Melcion (D.) et Devoyod (J. J.) (1974). - La flore microbienne du fromage de Cantal fabriqué à partir de lait cru. III. Rôle des levures dans la maturation de la "Tome ». Le Lait, 54 (539-540), 616.

[10] Santa Maria (J.) (1956). - Annales Inst. Nac. Invest. Agron., 5, 163. Cité par Lodder (J.) (1971), 332-334.

[11] Schmidt (J. L.) et Lenorr (J.) (1978). - Contribution à l'étude de la flore levure du fromage de Camembert. Son évolution au cours de la maturation (I). Le Lait, 58 (577), 355.

[12] Vergeade (J.), Guiraud (J.), Larpent (J. P.) et Galzy (P.) (1976). - Etude de la flore de levure du Saint-Nectaire. Le Lait, 56 (555-556), 275. 